\title{
Antimonides Type-II Superlattice Digital Focal Plane Arrays for Space Remote Sensing Instruments
}

Sarath Gunapala, David Ting, Alexander Soibel, Arezou Khoshakhlagh, Sam Keo, Sir Rafol, Anita Fisher, Cory Hill, Brian Pepper, Edward Luong, and Jason Mumolo

Center for Infrared Photodetectors, Jet Propulsion Laboratory, California Institute of Technology

Pasadena, California, USA

Sachidananda Babu and Parminder Ghuman

NASA Earth Science Technology Office

Greenbelt, Maryland, USA

Kwong-Kit Choi

US Army Research, Development and Engineering Command, Army Research Laboratory Adelphi, Maryland, USA

Arvind D'Souza and Christopher Masterjohn

DRS Network \& Imaging Systems, Inc.

Cypress, California, USA

October 9, 2018

International Conference on Space Optics

Chania, Greece 


\section{Outline}

- Barrier Infrared Detectors (BIRDs) technology

- Decrease detector dark current (i.e., reduce noise -> increase SNR)

- Resonator Pixel (RP) light coupling technology

- Increase detector quantum efficiency (i.e., increase SNR)

- 3D-Digital read out integrated circuits (DROICs) technology

- Increases the ROIC well depth (i.e., reduce noise -> increase SNR) 


\section{Outline}

- Barrier Infrared Detectors (Bİ̉Rs) technology

- Decrease detector dark ćurrent (i.ẹ., reduce noise -> increase SNR)

- Resonator Pixel (RP) light coupling technology

- Increase detector quantum efficiency (i.e., increase SNR)

- 3D-Digital read out integrated çircuits (DROICs) technology

- Increases the ROIC well depth (jóe., reduce noise -> increase SNR)

- Digital RP-BIRD focal plane arrays

- Increases SNR or increases operating temperature for same SNR

- Reduce the Size, Weight, and Power (SWaP) factor of the Integrated Detector Dewar Cooler Assembly (IDDCA) -> Enables

SmallSat applications

- MWIR imaging at $>200 \mathrm{~K}$; LWIR imaging at $>100 \mathrm{~K}$

- SF-400: 2W, 100W, 3.8Kg whereas SF-070: $800 \mathrm{~mW}, 30 \mathrm{~W}, 0.85 \mathrm{Kg}$

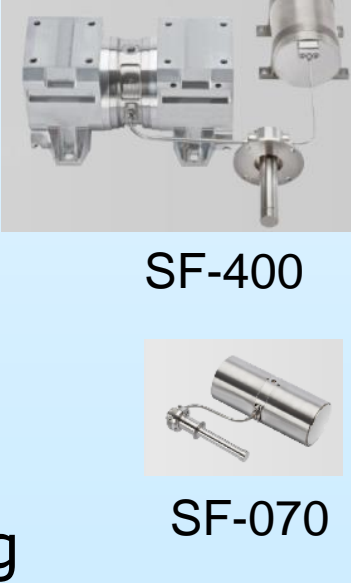




\section{Barrier Infrared Detector (e.g., nBn, XBn, CBIRD) Technology}


(a) p-n diode

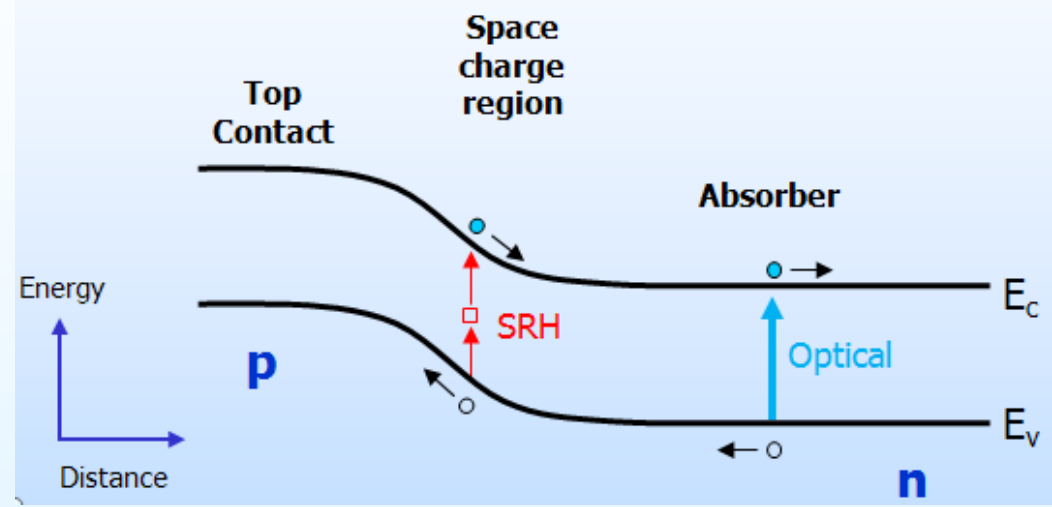

- Conventional p-n junction photodiode

- Suffers from generation-recombination (GR) dark current

- Primarily induced by mid-gap defects at the junction via Shockley-Read-Hall (SRH) processes

- Exponential process with activation energy of $E_{g} / 2$ (half of energy band gap)

- Also suffers from surface leakage dark current

- Resulting high dark current limits sensitivity and/or operating temperature (b)

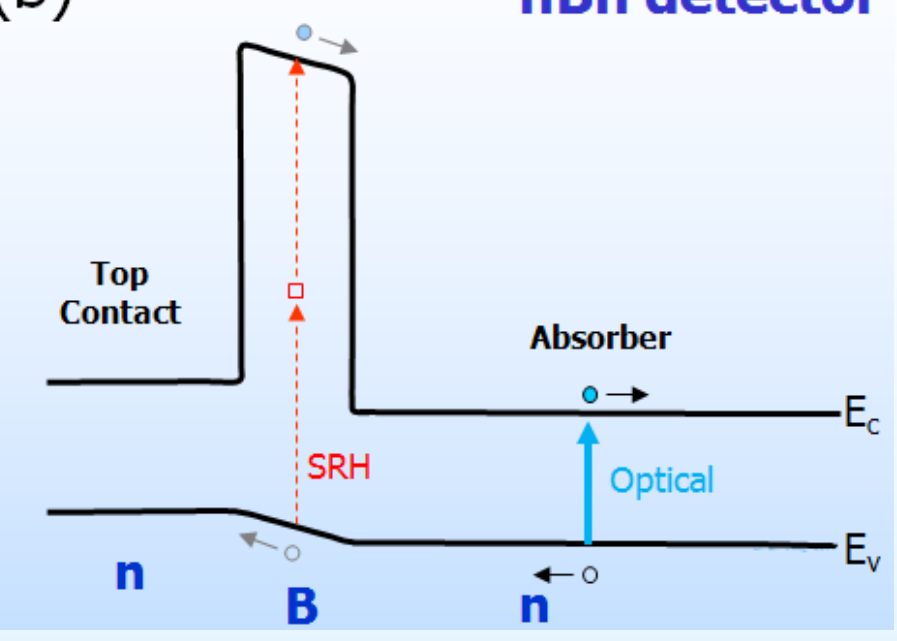

- $\quad$ HOT-BIRD based on the innovative $\mathrm{nBn}$ device architecture

- High band gap electron barrier inserted at the "junction"

- Blocking electrons but not holes

- Larger band gap of the barrier suppresses SRH processes and virtually eliminates GR dark current

- Barrier also serves to block surface leakage dark current

- Photocurrent flows un-impeded 


\section{InAs/GaSb vs. InAs/InAsSb T2SL}

(a) InAs/GaSb

\section{Material Growth Shutter Sequence}

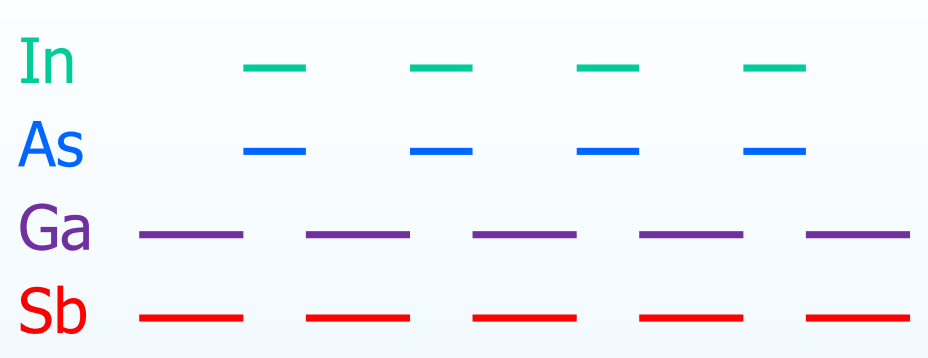

(b) InAs/InAsSb

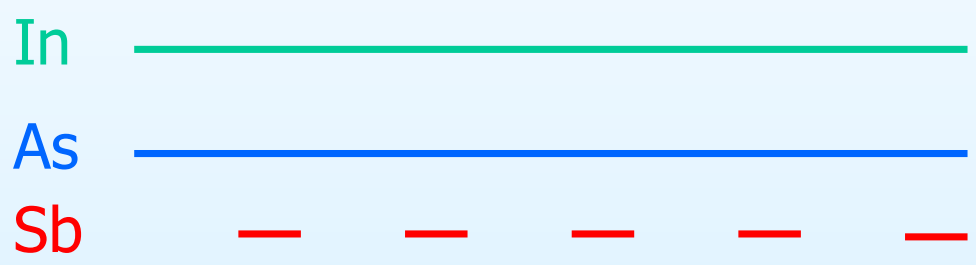

- InAs/InAsSb T2SLS growth is much simpler than InAs/GaSb T2SL

- 3 sources instead of 4 (no Gallium)

- 1 moving shutter instead of 4

- No complicated interface strain balancing

- Cutoff wavelength is easily adjusted

- InAs/InAsSb easy to grow and have good manufacturability 


\section{InAs/InAsSb Superlattice Material}

- InAs/InAsSb T2SLS (Ga-free SLS) absorber

- Band gap can be adjusted easily

- Covers cutoff wavelengths from $4 \mu \mathrm{m}$ to VLWIR

- Strain-balanced pseudomorphic growth on GaSb

- Material with different cutoff wavelength can be grown on the same substrate

- Long minority carrier lifetime

- To enable the construction of advanced heterostructure barrier infrared detectors (e.g., nBn, CBIRD) we also developed matching

- Electron unipolar barriers

- Hole unipolar barriers

- All grown on GaSb substrate

- Absorber, electron barrier, hole barrier can all be grade 


\section{GaSb Substrates}

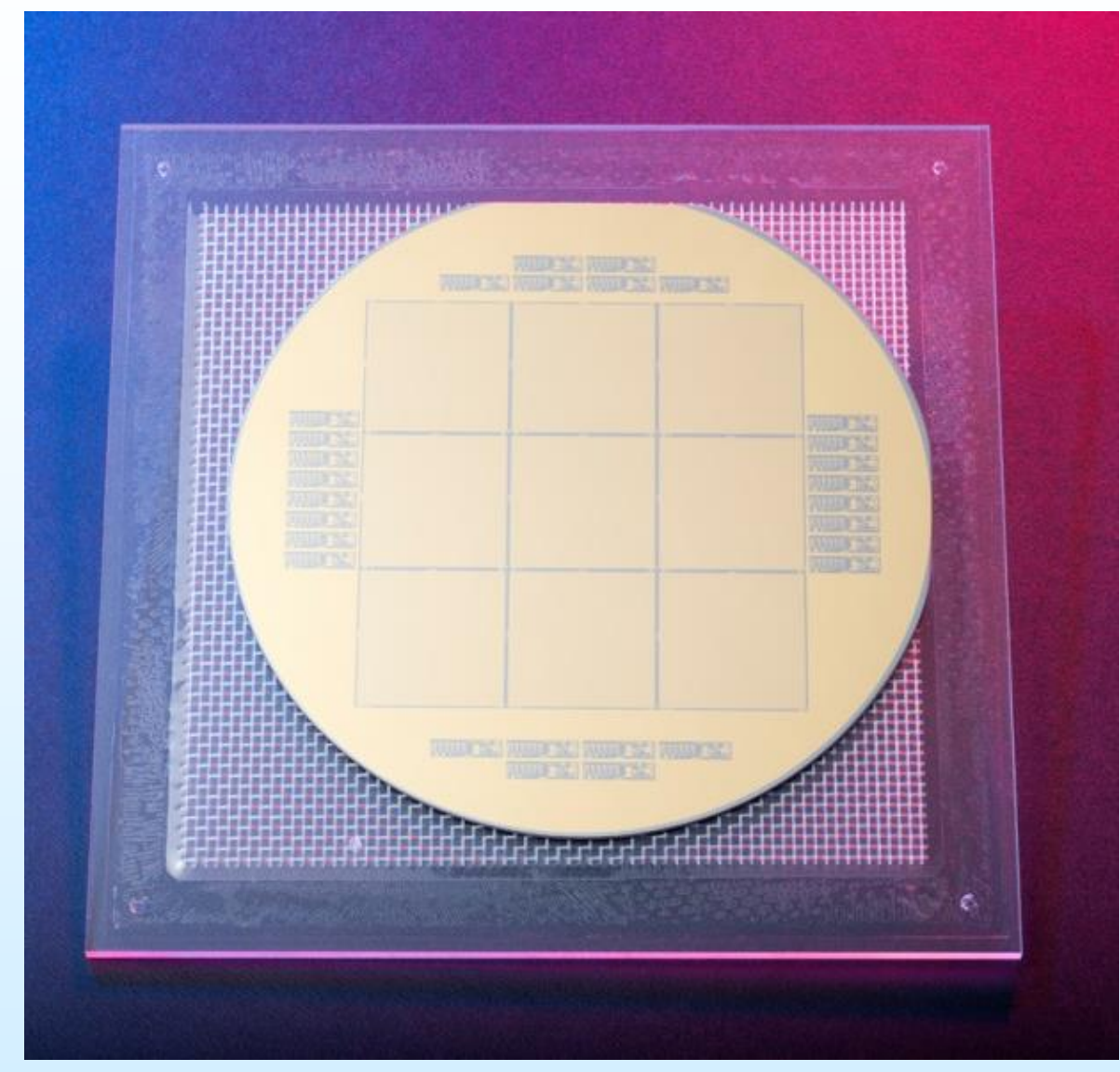

Nine $1 \mathrm{kx} 1 \mathrm{k}$ (19.5 $\mu \mathrm{m}$ pitch) detector arrays on 4-inch GaSb substrate.
- GaSb substrates available in $2^{\prime \prime}, 3^{\prime \prime}, 4^{\prime \prime}, 5^{\prime \prime}$, and 6" diameter formats

- Cost effective

- Multiple suppliers

- WaferTech

- Galaxy

- IntelliEPI

- Large area substrates

- Economy of scale

- Very large area arrays 

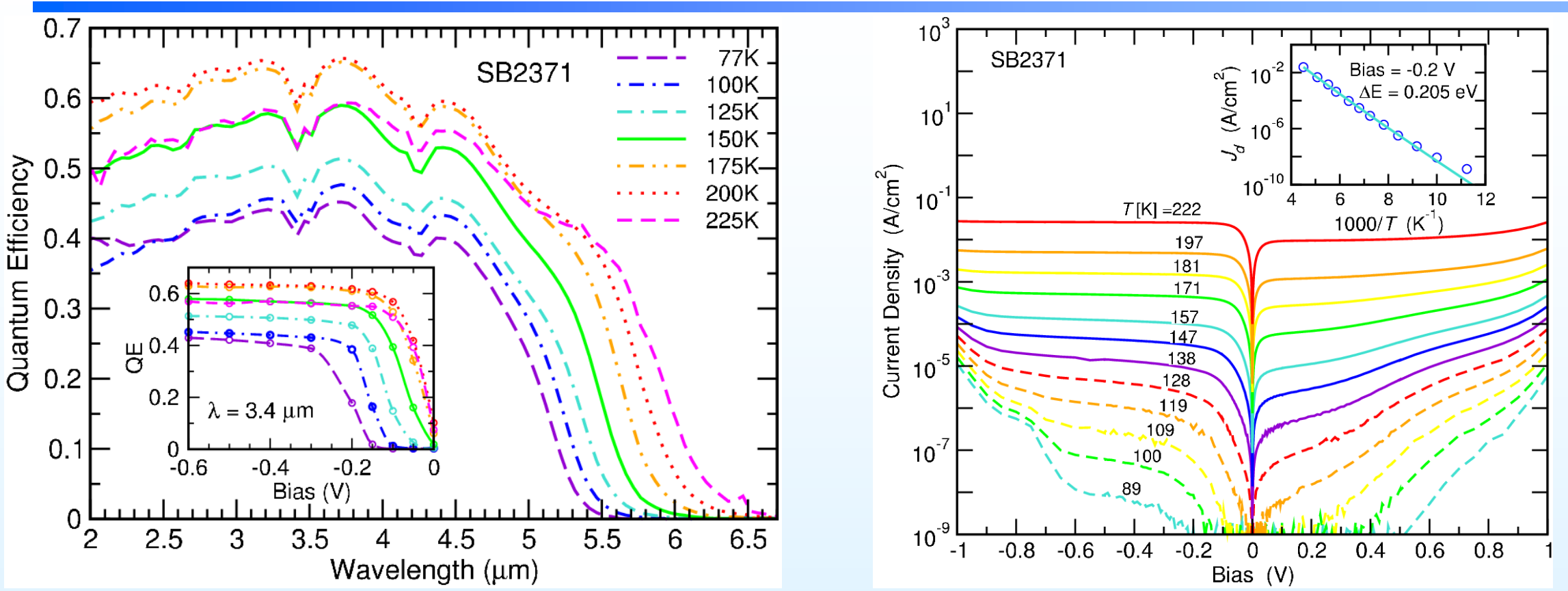

- MWIR nBn detector with InAs/InAsSb T2SLS absorber

- Cutoff wavelength: $5.07 \mu \mathrm{m}(77 \mathrm{~K}), 5.77 \mu \mathrm{m}(225 \mathrm{~K})$

- $\mathrm{QE}(4.3 \mu \mathrm{m}, 150 \mathrm{~K})=52 \%$ - No A/R coating

- $J_{\text {dark }}(-0.2 \mathrm{~V}, 157 \mathrm{~K})=9.6 \times 10^{-5} \mathrm{~A} / \mathrm{cm}^{2}$ ( $4.5 \mathrm{X}$ Rule'07)

- Arrhenius analysis ( $109 \mathrm{~K}$ to $222 \mathrm{~K}$ ):

- Activation energy $\Delta E=0.205 \mathrm{eV} ; \mathrm{Eg}(157 \mathrm{~K}) \sim 0.229 \mathrm{eV}$.

"Barrier InfraRed Detectors", D. Z. Ting, A. Khoshakhlagh, A. Soibel, Cory J. Hill, and S. D. Gunapala, U. S. Patent No. 8,217,480 (2012) 


\section{$\mathrm{NE} \Delta \mathrm{T}$ and Images}
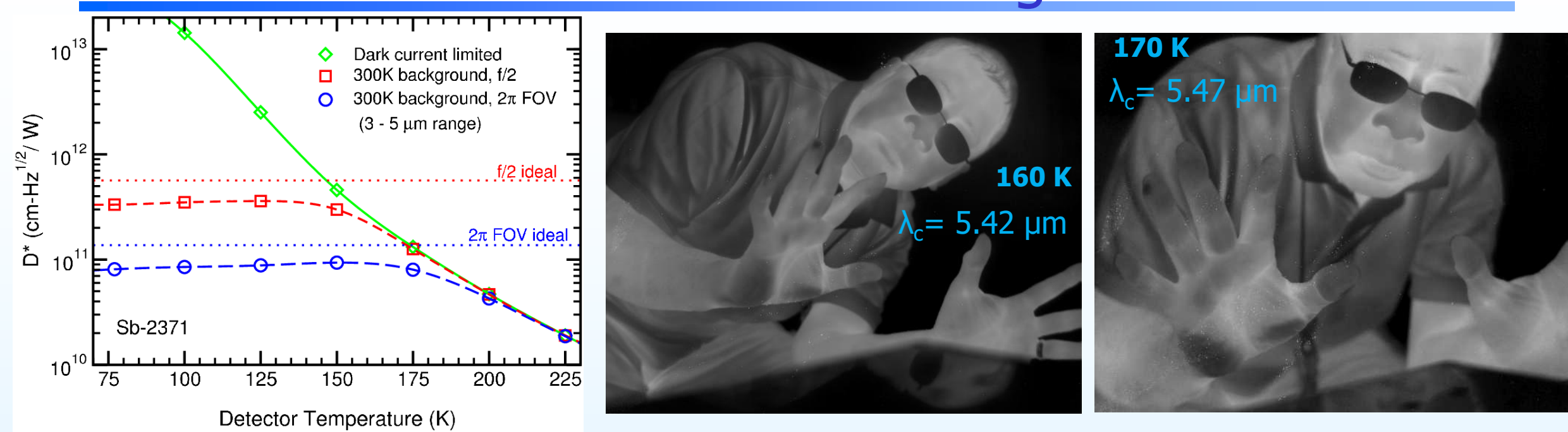

- Black-body $(3-5 \mu \mathrm{m}) \mathrm{D}^{*}$

- f/2: $D^{*}=3.4 \times 10^{11} \mathrm{~cm}-\mathrm{Hz}^{1 / 2} / \mathrm{W}$, BLIP at $152 \mathrm{~K}$

- Assuming $\mathrm{J}_{\text {dark }}=1 / 4 \mathrm{~J}_{\text {photo }}$

- FPA shows significantly higher operating temperature than InSb

- SBF-193 ROIC: $24-\mu m$ pitch, $640 \times 512$ format

- f/2 optics, 300K background

- 160K: NEAT 18.7 mK, Operability 99.7\%

- 170K: NEAT 26.6 mK, Operability 99.6\%

HOT-BIRD: "Mid-wavelength high operating temperature barrier infrared detector and focal plane array", D. Z. Ting, A. Soibel, A. Khoshakhlagh, S. B. Rafol, S. A. Keo, L. Höglund, A. M. Fisher, E. M. Luong, and S. D. Gunapala, Appl. Phys. Lett. 113 , 021101 (2018). doi: 10.1063/1.5033338 

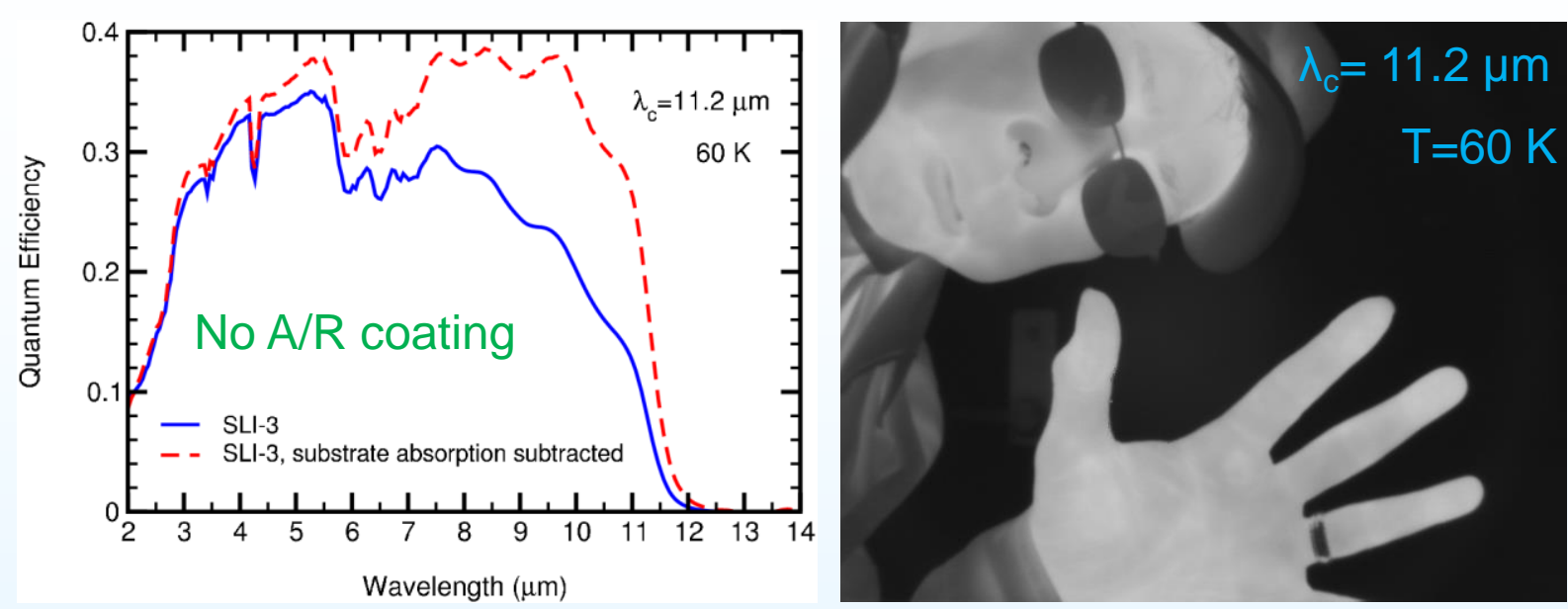

99.7\% operability (17SLL03)

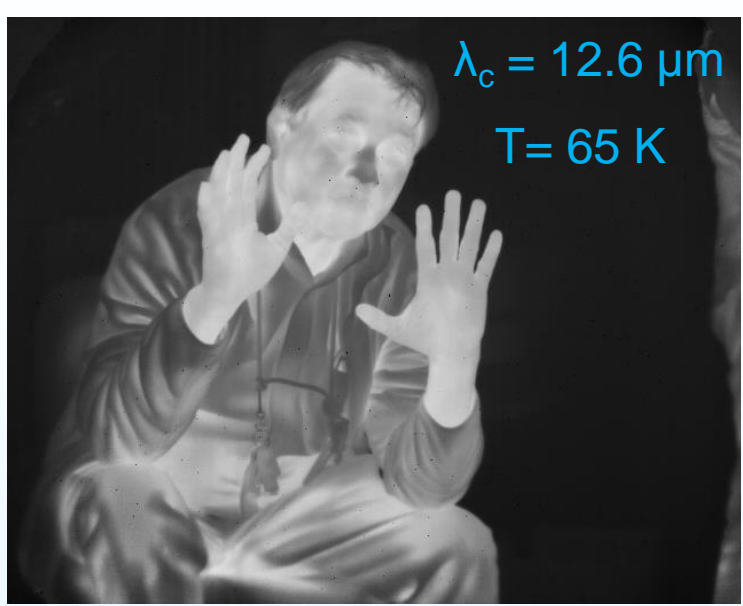

99.98\% operability (18SLL03)

- Developing T2SL-based LWIR detectors for NASA Sustainable Land Imaging Technology (SLI-T) Program

- Unipolar barrier infrared detector architecture, T2SL absorber

- High quality $\lambda_{\text {cutoff }} \sim 11.2 \mu \mathrm{m}$ T2SL absorber material

- 240 ns minority carrier lifetime

- $\mathrm{J}_{\text {dark }}(60 \mathrm{~K}) \sim 10^{-5} \mathrm{~A} / \mathrm{cm}^{2}$; QE 37\% without A/R coating.

- Very good FPA operabilities

- Also demonstrated $\lambda_{\text {cutoff }} \sim 12.6 \mu \mathrm{m}$ detectors/FPAs 


\section{Resonator Pixel Technology}




\section{Resonator-Pixel Technology}

\section{Concept:}

- Diffract incident light at an angle larger than the critical angle of total internal reflection to achieve threedimensional optical confinement.

- Use pixel active volume as a resonator to achieve coherent accumulation of light.
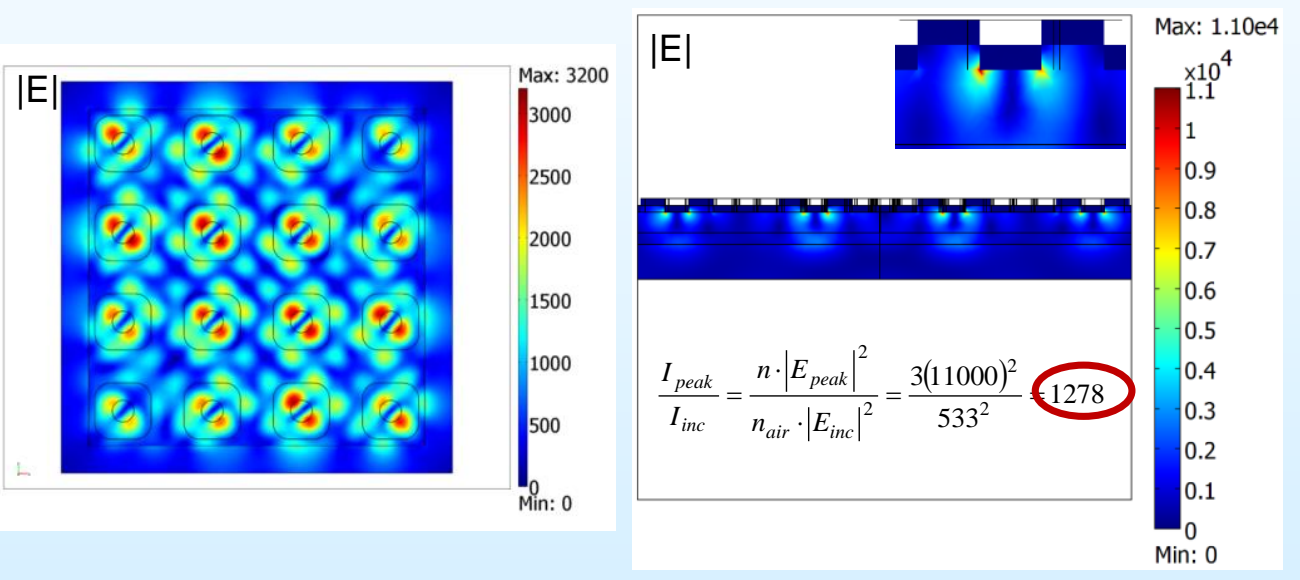

\section{Advantages:}

- Increases quantum efficiency

- Thin active layers (good for low carrier mobility)

- Low dark current (due to thin pixel)

- Free of anti-reflection coating 


\section{Digital Pixel Read Out Electronic Circuit (DROIC) Technology}




\section{Digital Read Out Integrated Circuits (DROICs)}

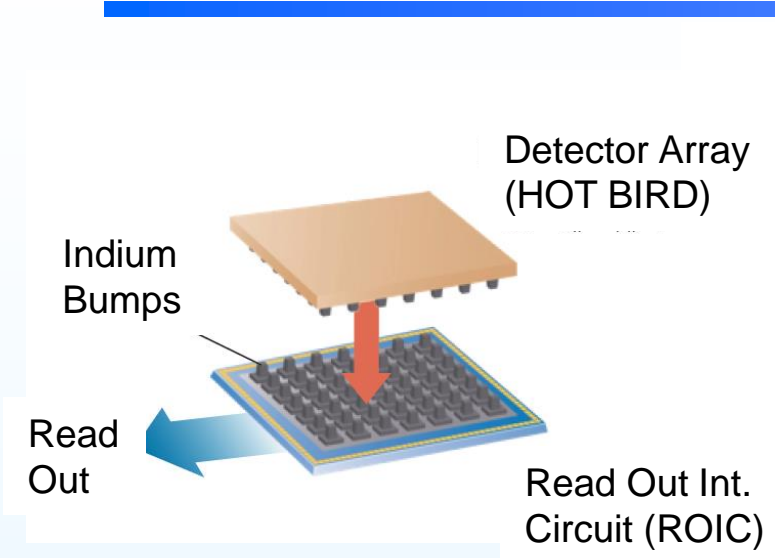

Circuit (ROIC)

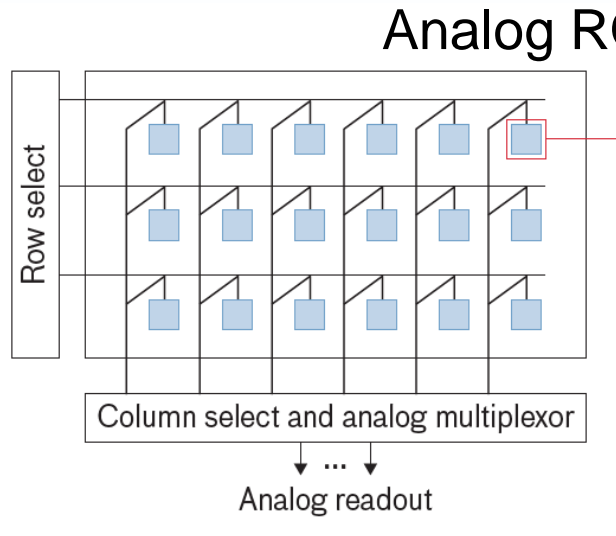

(a)

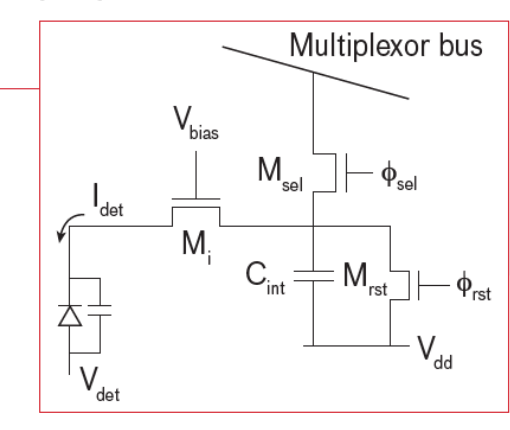

(b)

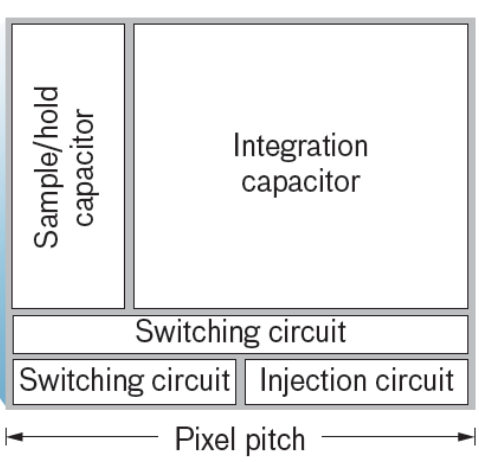

(c)

\section{Digital ROIC}

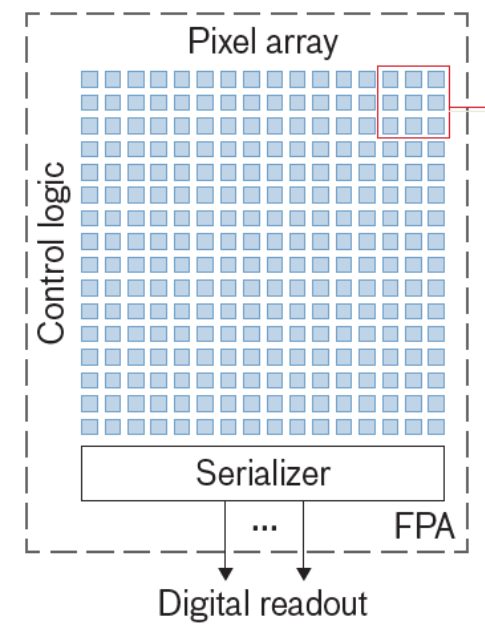

(a)

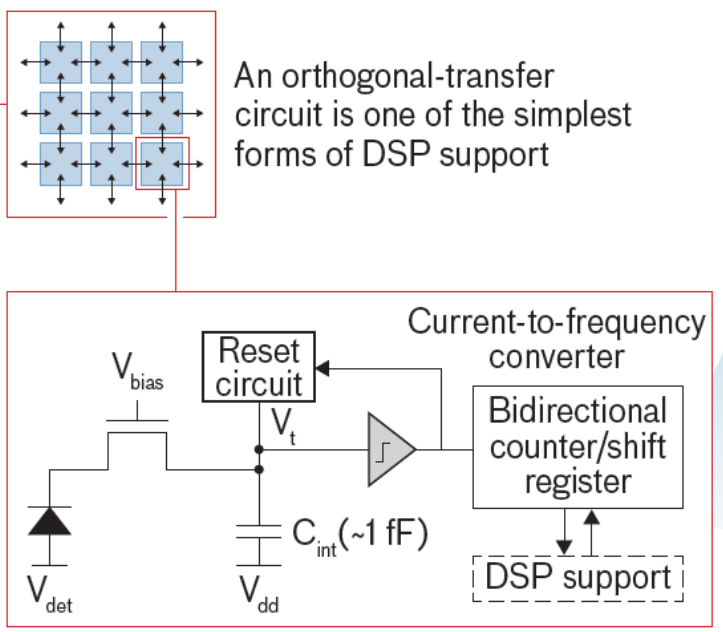

(b)
Analog current injection circuit and current-to-frequency converter

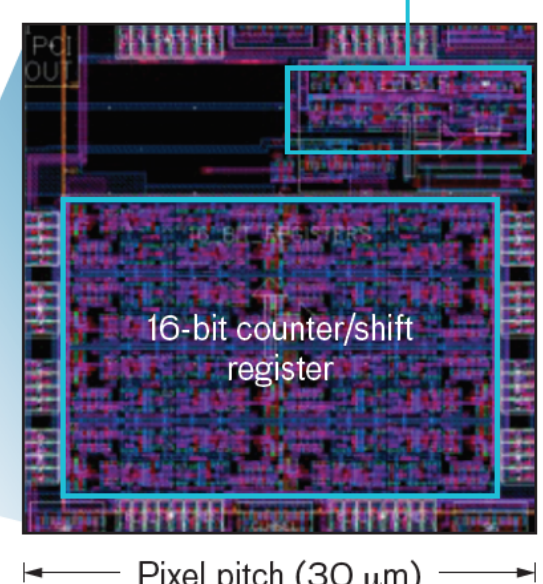

(c)

Ref: Kenneth I. Schultz, et al., "Digital-Pixel Focal Plane Array Technology", Lincoln Laboratory Journal, Vol. 20, Number 2 (2014). 
- Existing HyTES (Hyperspectral Thermal Emission Spectrometer) airborne instrument surveys $300 \mathrm{~K}$ background scene in the long-wavelength IR (LWIR) using QWIP FPA operating at $\sim 40 \mathrm{~K}$

- Assume we switch the FPA to Digital BIRD FPA

- $250 \mathrm{~K}$ scene (lower photon flux than 300K scene) $S N R_{\text {Max }}=\frac{\text { Signal }_{\text {Max }}}{\text { Noise }} \propto \sqrt{\text { Well Depth }_{\text {Max }}}$

- Cover bands in LWIR and mid-wavelength IR (MWIR; lower photon flux than LWIR)

- $65 \mathrm{~K}$ operation to reduce cryocooler size, weight and power (SWaP) for space flight

- But need to deal with much higher dark current level than at 40K

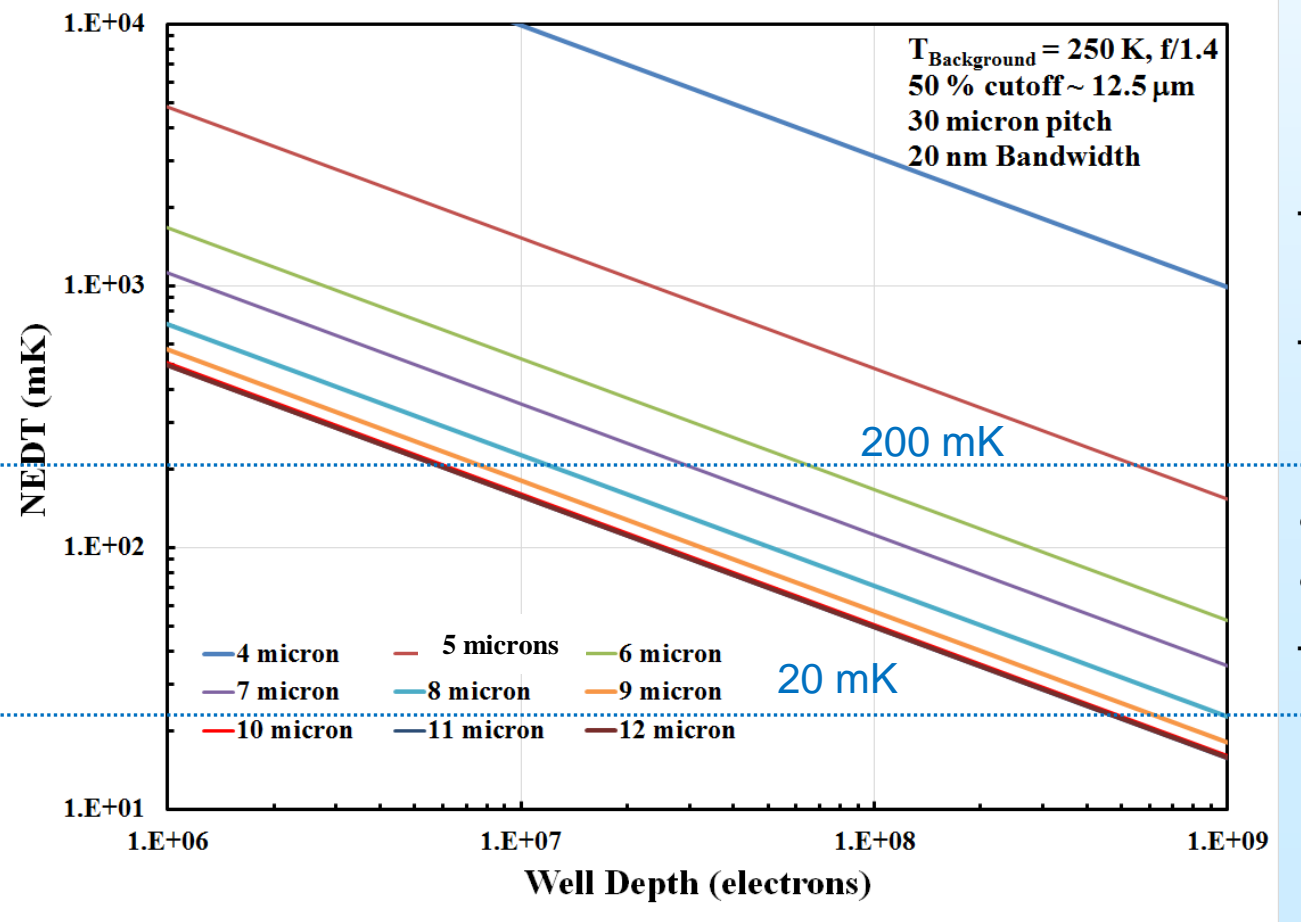

- Addressing challenges with Digital BIRD FPA

- BIRD for improved detector dark current and QE performance over QWIP

- Digital-pixel ROIC with large well depth enables much longer integration time to improve signal to noise ratio

- Conventional ROIC well depth $\sim$ few million e-'s

- D-ROIC well depth can exceed 1 billion $e^{-\prime} s$

- Can achieve $200 \mathrm{mK}$ NE$\Delta \mathrm{T}$ for $20 \mathrm{~nm}$ wide spectral band centered at $5 \mu \mathrm{m}$ (\& longer)at $65 \mathrm{~K}$ only with D-ROIC large well depth 
- TIRS instrument on Landsat 8 surveys $300 \mathrm{~K}$ background scene in the longwavelength IR (LWIR) using QWIP FPA operating at $\sim 43 \mathrm{~K}$

- Assume we switch the FPA to Digital BIRD FPA

- $250 \mathrm{~K}$ scene (lower photon flux than 300K scene)

- Cover bands Landsat LWIR bands ( $0.5 \mu \mathrm{m}$ wide bands)

- $65 \mathrm{~K}$ operation to reduce cryocooler size, weight and power (SWaP) for space flight

- But need to deal with much higher dark current level than at $40 \mathrm{~K}$

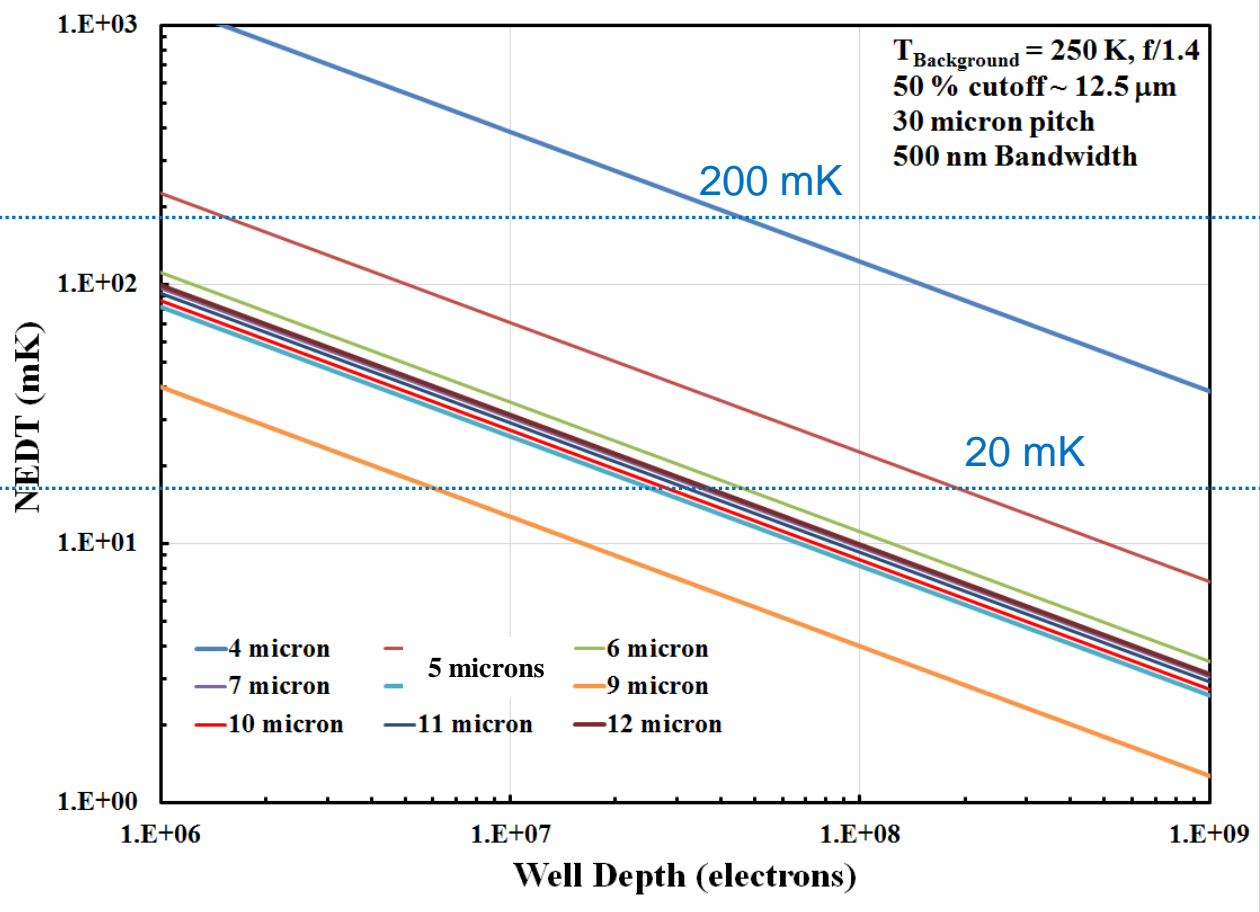

- Addressing challenges with Digital BIRD FPA

- BIRD for improved detector dark current and QE performance over QWIP

- Digital-pixel ROIC with large well depth enables much longer integration time to improve signal to noise ratio

- Conventional ROIC well depth $\sim$ few million e-'s

- D-ROIC well depth can exceed 1 billion $e^{-\prime} s$

- Can achieve $20 \mathrm{mK} \mathrm{NE} \Delta \mathrm{T}$ for $500 \mathrm{~nm}$ wide spectral band centered at $5 \mu \mathrm{m}$ (\& longer) at $65 \mathrm{~K}$ only with $\mathrm{D}$-ROIC large well depth 


\begin{tabular}{|c|c|c|c|c|c|}
\hline ROIC & $\begin{array}{c}\text { ISC-9803 } \\
\text { AROIC }\end{array}$ & $\begin{array}{c}\text { ISC-0905 } \\
\text { AROIC }\end{array}$ & DRS 3D-DROIC & DRS 3D-DROIC & DRS 3D-DROIC \\
\hline Binning & $1 \times 1$ & $1 \times 1$ & $1 \times 1$ & $3 \times 3$ & $4 \times 4$ \\
\hline ROIC Format & $640 \times 512$ & $640 \times 512$ & $2004 \times 2008$ & $668 \times 668$ (eff.) & $501 \times 502$ (eff.) \\
\hline Pixel Pitch & $25 \mu \mathrm{m}$ & $30 \mu \mathrm{m}$ & $8 \mu \mathrm{m}$ & $24 \mu \mathrm{m}$ (eff.) & $32 \mu \mathrm{m}$ (eff.) \\
\hline Well Depth & $11 \mathrm{Me}^{-}$ & & & $e^{-}$(leff.) & $2,780 \mathrm{Me}^{-}$(eff.) \\
\hline
\end{tabular}

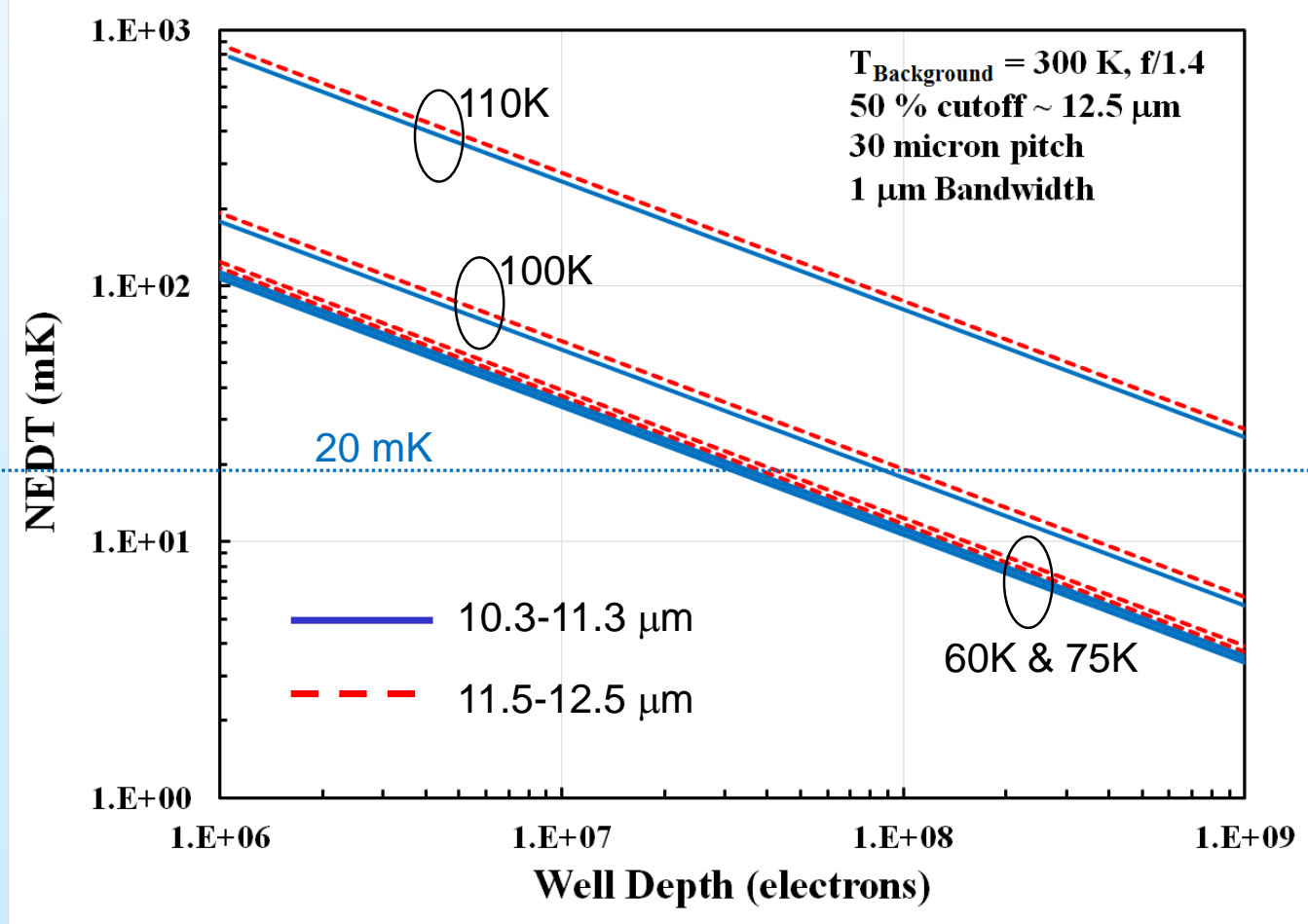

- Addressing challenges with Digital BIRD FPA

- BIRD for improved detector dark current and QE performance over QWIP

- Digital-pixel ROIC with large well depth enables much longer integration time to improve signal to noise ratio

- Conventional ROIC well depth $\sim$ few million $e^{-\prime} s$

- D-ROIC well depth can exceed 1 billion e-'s

- Can achieve 20 mK NE $\Delta T$ for 500 nm wide spectral band centered at $10-12.5 \mu \mathrm{m}$ only with D-ROIC large well depth 


\section{Summary}

- Recent advances in InAs/InGaAs Barrier IR Detector (BIRD) technology is a breakthrough

- Elevated the FPA operating temperature, good uniformity \& operability, and good manufacturability

- MWIR for CIRAS mission

- LWIR for HyTI mission

- Resonator Pixel technology

- Will increase quantum efficiency

- Digital ROIC is a breakthrough technology

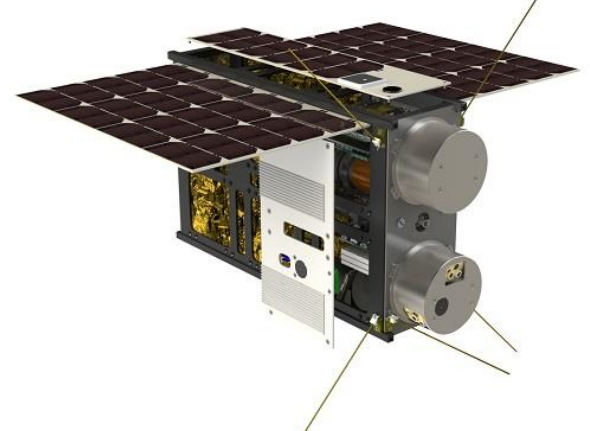

Image Credit: https://n-avionics.com/

- Elevates operating temperature

- RP-BIRD DFPA elevates the operating temperature of FPAs

- Enables the low cost Cubesats \& Smallsats (for IR land imaging, Spectrometers, and sounders), Lower SWaP factor

- $100 \mathrm{~K}$ for LWIR for land imaging

- This work is sponsored by NASA ESTO under ACT \& SLI-T programs 